\title{
IMPROVED PHOTOELECTROTHERMAL MODEL WITH THERMAL PARAMETERS VARIATION APPLIED TO AN EXTRA-HIGH CURRENT COB LED
}

\author{
Dênis de C. Pereira ${ }^{1}$, Pedro L. Tavares ${ }^{1}$, Pedro S. Almeida ${ }^{1}$, Guilherme M. Soares ${ }^{1}$, Fernando L. Tofoli ${ }^{2}$, \\ Henrique A. C. Braga ${ }^{1}$ \\ ${ }^{1}$ Federal University of Juiz de Fora (Modern Lighting Research Group - NIMO), Juiz de Fora - Minas Gerais, Brazil \\ ${ }^{2}$ Federal University of São João del-Rei, São João del-Rei - Minas Gerais, Brazil \\ e-mail: denis.castro@engenharia.ufjf.br
}

\begin{abstract}
This work is mainly concerned with the improved photoelectrothermal (IPET) modeling of an extra-high current (EHC) and extra-high luminous flux (up to $60 \mathrm{klm}$ ) solid-state light source based on a chip-onboard (COB) light-emitting diode (LED). The studied COB technology presents the particular challenge of extremely small thermal resistances, with extra-high current levels through the lamp (up to $12 \mathrm{~A}$ ). Considering the unique thermal characteristics of such devices, an improved PET modeling and its respective experimental methodology are detailed. Accurate techniques are also proposed to estimate the device junction temperature. The studied method is very suitable to represent EHC COB devices, since it includes the main thermal parameters variation into a novel model, optimizing the flux prediction for this technology. In this context, the static flux and efficacy are analyzed by means of experimental tests and theoretical model, where the improvement of the employed method is highlighted.
\end{abstract}

Keywords - Extra-high current COB LEDs, Floodlighting, Improved photoelectrothermal modeling, Thermal parameters variation.

\section{INTRODUCTION}

Nowadays, solid-state lighting is a subject of great interest for both academia and industry. In this scenario, lightemitting diode (LED) technology is currently established as a prominent solution for distinct applications in indoor or outdoor environments due to its remarkable characteristics regarding efficiency, wide variety of models with different power spectral distribution (PSD), high luminous efficacy, long lifespan, high reliability, and environmental friendliness [1]. Typically, for large areas that require expressive high luminous flux levels, most applications are based on series association of discrete LED chips, resulting in a high-voltage low-current module [2]. More recently, the chip-on-board (COB) technology involving high currents and low voltage levels has also been considered due to its intrinsic thermal advantages respected to several chip topologies, bringing new challenges to the lighting field [3].

Regarding physical concepts, LEDs can be arranged on a single-substrate compact matrix structure called COB. High-

Manuscript received 09/12/2018; first revision 24/01/2019; accepted for publication 15/02/2019, by recommendation of Editor Marcello Mezaroba. http://dx.doi.org/10.18618/REP.2019.2.0054 powered COB LED lighting applications are aimed at sport stadiums and courts, airport runways, international borders, maritime and mining environments, which require extra-high luminous flux levels. In this work, an extra-high current (EHC) COB LED from Flip Chip Opto (FCOpto) is employed. This manufacturer explores commercially the Apollo and Luna series, which can perform high luminous flux (up to 230,000 $\mathrm{lm}$ ) and innovative extra-high current levels (up to 48 A) [4]. Thus, Apollo 600 EHC COB model [5] is analyzed in detail, while its lighting performance is carefully pointed out and discussed.

When dealing with the well-known low-current discrete LEDs or even some popular COB LEDs (restricted to low rated currents [6], [7]), most of the datasheets are usually adequate and comprehensive, which describe essential parameters to perform a concise photoelectrothermal (PET) characterization [8]. On the other hand, information regarding $\mathrm{EHC}$ COB LEDs as provided in datasheets is normally incomplete and restricted to very few manufacturers, in such a way a thorough analysis must be carried out in this case [9], [10]. Therefore, a contribution of this work lies in the detailed and straightforward description of each one of the experimental PET curves, which are adequate to improve EHC COB LEDs technical datasheets.

The modeling methods described in literature are wellknown for low-current discrete devices [11]-[13]. Thus, this work introduces an improved model for the EHC COB technology, while also proposing accurate methodologies to predict its junction temperature variation so that important thermal aspects can be incorporated into a specific and accurate model. The variations in thermal-related parameters when operating under high current and high junction temperature conditions are critical in the COB technology to establish a reliable model, which properly attenuates the flux curve above nominal current levels. Therefore, such thermal variations are addressed from experimental tests and properly considered into a novel improved PET (IPET) model.

The paper is organized as follows. Section II presents the state-of-the-art regarding the EHC COB technology, while its main characteristics are highlighted. Section III describes a reproducible experimental methodology for the complete static characterization of EHC COB LEDs. Moreover, a novel improved model is also presented and discussed, which considers the heat dissipation factor and junction resistance variations in the device for an accurate fitting of the obtained curves with the ones extracted experimentally. An experimental analysis is used to validate the derived model in 
Section IV, with good accuracy and minimal errors at very high current ratings. Besides, a theoretical heatsink is estimated based on the improved model, while the feasibility of the employed technique is shown. Finally, relevant aspects are discussed and the main contributions of this paper are summarized in Section V.

\section{EXTRA-HIGH CURRENT COB LEDS}

Several series or parallel/series-connected LED-based luminaires have been proposed considering the association of discrete elements, thus requiring a high-voltage module to obtain higher output power levels as a consequence. On the other hand, aiming at volume and size minimization, modern high-power $\mathrm{COB}$ devices are designed featuring low-voltage and extra-high current levels [4], [5]. The analysis carried out in this work considers the EHC COB model Apollo 600, which is shown in Figure 1. It can be seen that the main diameter considering the heatsink is equal to $341 \mathrm{~mm}$ with a weight of $6.36 \mathrm{~kg}$, which represents a significant reduction when compared to traditional associations of discrete elements to achieve high voltage levels [14].

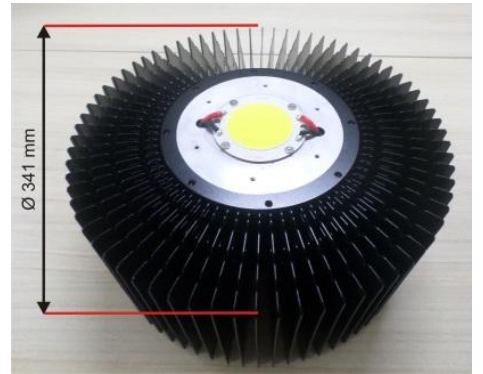

Fig. 1. EHC COB LED Apollo 600 mounted on a $600-\mathrm{W}$ aluminum heatsink.

The COB technology consists of a miniaturized LED chip matrix mounted on a substrate or circuit board. This design provides higher power density and uniform light output [3]. Considering extra-high current levels through the $\mathrm{COB}$ device, the matrix composed by LEDs must be properly mounted on a single substrate, whereas an improved thermal management is extremely relevant. Considering the EHC COB LEDs from FCOpto, high-power devices are conceived through a 3-pad patented technology, which is presented in Figure 2.a. This mounting technique offers extra-low thermal resistance and better thermal dissipation through a pillar structure, which allows extra-high current/luminous flux operation and brings new challenges to the lighting field. From Figure 2.a, instead of the regular flip-chip LED, the 3pad flip-chip presents an additional thermal-pad (T-pad), with improved heat dissipation from the metal core printed circuit board. Figure 2.b shows the main dimensions of the EHC COB device, while Table I lists the main characteristics regarding Apollo 600 [5].

\section{TABLE I}

Parameters Obtained From Apollo 600 Datasheet

\begin{tabular}{cccc}
\hline Parameter & Symbol & Value & Unit \\
\hline Maximum power dissipation & $P_{\max }$ & 608.4 & $\mathrm{~W}$ \\
\hline Maximum dc forward current & $I_{C O B(\max )}$ & 12 & $\mathrm{~A}$ \\
\hline Nominal dc forward current & $I_{0}$ & 6 & $\mathrm{~A}$ \\
\hline Maximum luminous flux @ $12 \mathrm{~A}, 25^{\circ} \mathrm{C}$ & $\Phi_{\max }$ & 60840 & $1 \mathrm{~m}$ \\
\hline Maximum junction temperature & $T_{j(\max )}$ & 140 & ${ }^{\circ} \mathrm{C}$ \\
\hline Correlated color temperature & $\mathrm{CCT}$ & 5000 & $\mathrm{~K}$ \\
\hline
\end{tabular}

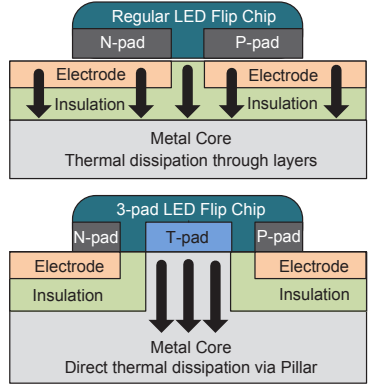

(a)

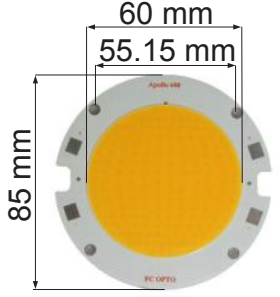

(b)
Fig. 2. (a) 3-pad chip integration technology and (b) Apollo 600 main dimensions [5].

\section{METHODOLOGY FOR STATIC PHOTOELECTROTHERMAL MODELING}

The static PET analysis comprises an investigation of the system composed by the COB, heatsink, and environment, also considering the thermal steady-state operation and the power supply at a constant dc current level, while also neglecting ac ripple levels. Thus, it is necessary to properly correlate the intrinsic thermal, electrical, and photometrical characteristics of the studied device. In this context, the theoretical waveforms regarding the static characterization are presented in Figure 3, where $T_{0}$ represents the reference temperature and $I_{0}$ is the reference current, which can be properly found in datasheet. Therefore, these curves must be experimentally described to determine all parameters required for the complete static modeling.
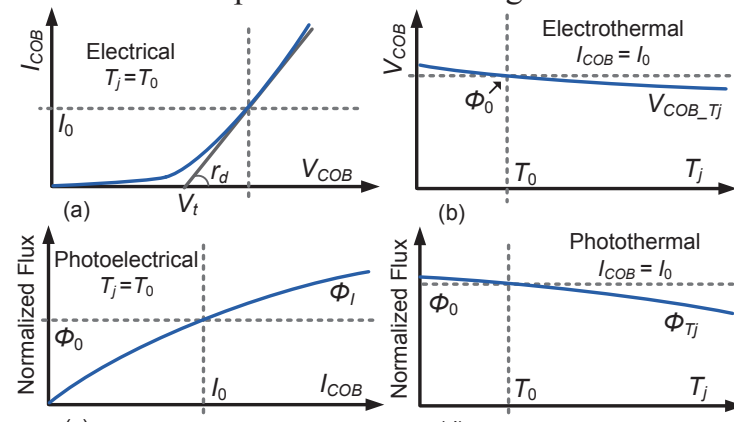

(c)

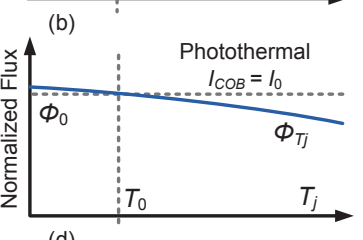

(d)

Fig. 3. Typical photoelectrothermal curves for the studied EHC COB LED. (a) Electrical curve, (b) electrothermal curve, (c) photoelectrical curve and (d) photothermal curve.

\section{A. Electrothermal Basics}

Considering Figure 3.a and operation above the threshold point, the equivalent EHC COB LED electrical model consists of a dynamic resistance $r_{d}$ and a threshold voltage drop $V_{t}$ in series with an ideal diode. This idealized model is given by (1), whereas the involved parameters do not vary with temperature.

$$
V_{C O B}=r_{d} \cdot I_{C O B}+V_{t}
$$

where $V_{C O B}$ is the forward voltage and $I_{C O B}$ is the current through the COB device.

In order to plot the electrical characteristic, the respective $I \times V$ (current versus voltage) curve must be obtained at the datasheet reference temperature $T_{0}=25^{\circ} \mathrm{C}$. Firstly, the ambient temperature $T_{a}$ is monitored by a thermo-hygrometer while the device case temperature $T_{c}$ is equally monitored by a thermocouple. In this case, the key parameter is the device junction temperature $T_{j}$, which must be maintained close to 
the reference value $T_{0}$. Although $T_{j}$ is not usually accessible for direct measures, the proper control of ambient and case temperatures ensures that the EHC COB LED temperaturedependent effect will not impact on this characterization. Thus, the ambient temperature is properly maintained at $T_{0}$ (using a heater or air conditioning system), while only a short current pulse (12 A rated up to 2 milliseconds) is applied in order to avoid temperature variations between $T_{c}$ and $T_{j}$. Since the device is always off, except during the current pulse application, no thermal flux comes from the chips themselves, thus resulting in $T_{0} \cong T_{a} \cong T_{c} \cong T_{j}$. The obtained voltages and currents are then correlated under approximately constant junction temperature for all the measured points as seen in Figure 4, which represents the electrical model for Apollo 600. From this curve, parameters $V_{t}=40.5 \mathrm{~V}$ and $r_{d}=0.95 \Omega$ can be defined.

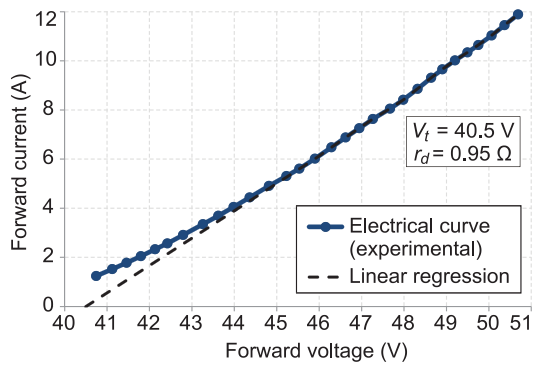

Fig. 4. Experimental electrical characterization for Apollo 600 $\left(T_{0}=25^{\circ} \mathrm{C}\right)$.

According to the electrothermal curve presented in Figure 3.b, temperature effects can be taken into account and incorporated to the model. As it was previously stated, the threshold voltage decreases as the temperature increases. Such characteristic associated to a negative temperature coefficient as presented by low-current discrete devices is a key parameter that must be properly analyzed. However, since in many datasheets of EHC COB LEDs this parameter is not given, the thermal voltage coefficient must be determined experimentally. The electrothermal model is described in (2), which relates the thermal voltage coefficient $k_{v}$ and the junction temperature $T_{j}$ so that it is possible to evaluate the real threshold voltage.

$$
V_{C O B}\left(I_{C O B}, T_{j}\right)=r_{d} \cdot I_{C O B}+\left[V_{t}+k_{v}\left(T_{j}-T_{0}\right)\right] \text {. }
$$

In order to obtain parameter $k_{v}$, an experiment has been implemented by placing and analyzing the studied device inside climatic chamber model WEISS WKL-100/40 [15]. Thus, this experiment evaluates the temperature effect on the threshold voltage, which consists in obtaining several $I \times V$ curves related to several points of junction temperature. Also in this case, a short current pulse (up to 2 milliseconds) must also be applied, while the junction temperature will not be significantly modified according to the chamber temperature, which must be previously set as desired.

The first $I \times V$ curve is obtained close to the ambient temperature, i.e., $25^{\circ} \mathrm{C}$, while the subsequent ones are generated considering steps of $5^{\circ} \mathrm{C}$. At each measurement, the thermal steady-state must be assumed before pulse the current, since the thermal time constants are much larger than the pulse width itself. The obtained curve is known as electrothermal curve, which can be seen in Figure 5. Thus, coefficient $k_{v}$ can be found to be $-25.4 \mathrm{mV} /{ }^{\circ} \mathrm{C}$, i.e., the angular coefficient extracted from the linear fitting curve.

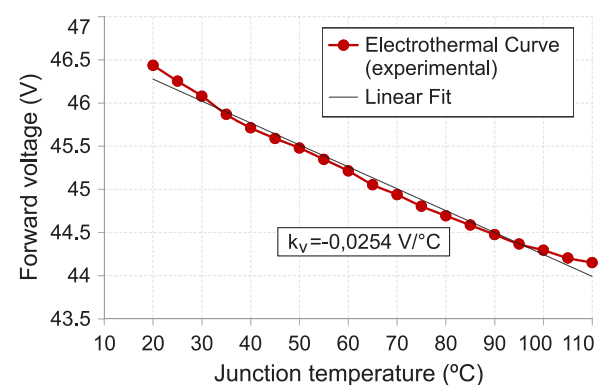

Fig. 5. Experimental electrothermal characterization for Apollo 600 $\left(I_{0}=6 \mathrm{~A}\right)$.

According to [16], the dynamic resistance variation does not present significant impact on the characteristics of lowcurrent discrete LED devices. Therefore, this parameter is often neglected, since the resistance can be considered constant over a wide range of temperature values. In order to ensure that the variation of $r_{d}$ does not influence the studied EHC COB device significantly, the previous $I \times V$ curves and their respective dynamic resistances have been also analyzed for several temperature levels. The resulting curve is shown in Figure 6, which represents the dynamic resistance behavior as a function of the junction temperature. From this analysis, $r_{d}$ varies slightly with temperature (from $0.95 \Omega$ at $25^{\circ} \mathrm{C}$ to $0.85 \Omega$ at $100{ }^{\circ} \mathrm{C}$ ), as it can be considered constant in the static modeling. Moreover, the average percent difference of the luminous flux corresponds to only $0.046 \%$ when considering such minimal variation.

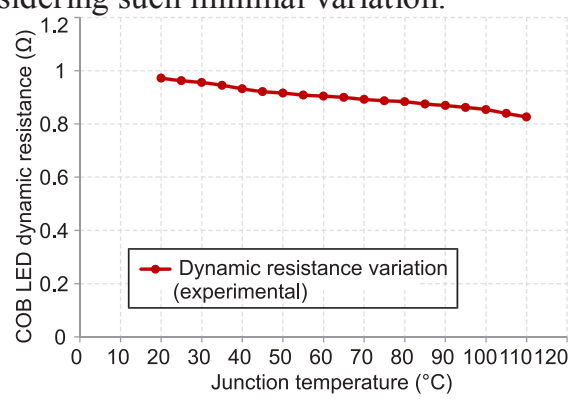

Fig. 6. Dynamic resistance electrothermal variation for Apollo 600.

In the electrothermal characterization, the heat dissipation effect is included to derive the related model. The equivalent electrothermal circuit of an EHC COB LED mounted on a typical heatsink is represented in Figure 7. Usually, in order to consider the thermal effect in the system, a thermaldomain circuit model is used. The dissipated thermal power $Q_{t h}$ can be obtained from(3), which corresponds to part of the total power delivered to the device. Not all power delivered to the device is converted into luminous radiation, as $P_{d}$ is the total power dissipation and coefficient $k_{h}$ represents the portion that is converted into heat, i.e., the heat dissipation factor. The radiant efficiency is then obtained by (4), while the heat dissipation factor corresponds to its complementary value.

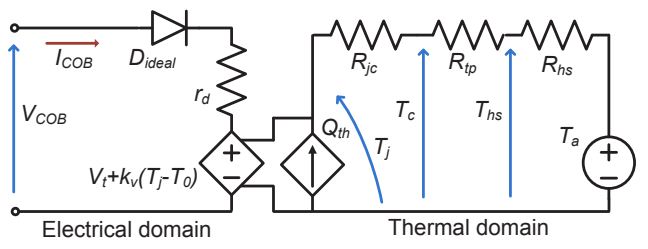

Fig. 7. Approximate electrothermal circuit for an EHC COB LED mounted on a heatsink. 


$$
\begin{gathered}
Q_{t h}=k_{h} \cdot I_{C O B} \cdot V_{C O B} \\
\eta_{r a d}=\frac{P_{r a d}}{P_{d}}, k_{h}=1-\eta_{\text {rad }}
\end{gathered}
$$

where $\eta_{\text {rad }}$ is the radiant efficiency, $P_{\text {rad }}$ is the radiant power and $P_{d}$ is the EHC COB LED dissipated power.

The thermal resistance between the junction and case is given by $R_{j c}$, which is usually found in the device datasheet (i.e., $0.008^{\circ} \mathrm{C} / \mathrm{W}$ for Apollo 600). When considering discrete LED associations, several discrete devices are placed on the same heatsink, whereas an equivalent thermal circuit should represent the parallel association of several power sources in series with their respective thermal resistances. Otherwise, when analyzing a single COB element, miniaturized LED associations are performed considering a single substrate. Such mounting technique facilitates the determination of a single equivalent junction-to-case thermal resistance value, which represents the LEDs internal association and simplifies the model.

At this point, it is also important to notice that extremely small values are featured for $R_{j c}$ when dealing with modern high power EHC COB devices. This characteristic impacts significantly on their performance, since it enables the $\mathrm{COB}$ LED to handle extra-high current levels without considerable thermal degradation. Additionally, with very small $R_{j c}$ values, any minimal variation influences the device model significantly, while this work also considers the variations in such parameter. Similarly, in order to improve the thermal performance, a heatsink with extra-small thermal resistance must be also employed. Considering Apollo 600, the employed heatsink (for power levels up to $600 \mathrm{~W}$ ) presents thermal resistance $R_{h s}=0.0785^{\circ} \mathrm{C} / \mathrm{W}$ [17].

\section{B. EHC COB LED Thermal Parameters Variation}

The junction temperature indirect determination has been studied in [18], [19] and [20], as this parameter is not experimentally estimated, but calculated by means of other parameters. In summary, the junction temperature can be estimated from (5) while rearranging (2) in order to obtain $T_{j}$. This indirect technique assumes that the voltage across the device $p-n$ junction varies with temperature, so that it is compared with the respective voltage at ambient temperature reference condition [18]. Therefore, another $I \times V$ curve must be plotted, also considering the thermal lighting degradation in this case, which is presented in Figure 8. A period of 30 minutes has been considered before each consecutive measurement to ensure proper thermal stabilization of the EHC COB LED device. It is important to highlight that expression (5) considers only electrical parameters, which avoids the $R_{j c}$ previous information in order to estimate $T_{j}$. Thus, the related junction thermal resistance can be consequently found from (6).

$$
T_{j}=T_{0}+\frac{V_{C O B}-V_{0}}{k_{v}}
$$

where $V_{C O B}$ and $V_{0}$ are the EHC COB LED voltage under test condition and reference ambient temperature condition, respectively.

$$
R_{j c}=\frac{T_{j}-T_{a}}{k_{h} \cdot P_{d}}-R_{h s} .
$$

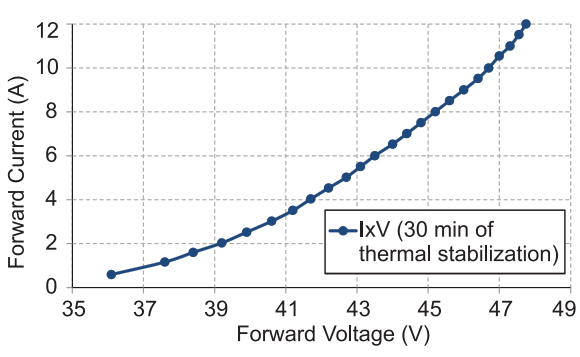

Fig. 8. Electrothermal $I \times V$ behavior for Apollo 600 with thermal stabilization time of 30 min between consecutive measurements.

In this work, an approximate experimental estimation of the junction temperature is proposed and compared to the literature indirect technique, while the test setup is presented in Figure 9. Basically, a temperature-controlled room is used while the EHC COB LED junction temperature is estimated with a thermal camera after the device thermal stabilization.

In order to calibrate the thermal camera, this work has employed a Teflon special tape with well-known emissivity, i.e., 0.92, which must be stuck at the measurement point and properly framed along with the screenshots. By performing this procedure, the thermal camera will recognize both the EHC COB LED and Teflon tape surfaces, being responsible for calibrating the instrument while estimating the device junction temperature in an approximate way. For low-current discrete LEDs, their own surfaces emissivities have also been considered in [21]. Otherwise, in this work, the EHC COB LED surface emissivity is not surely known, especially because this is a very modern device. Therefore, the Teflon tape calibration method has been used due to its industrial well-known emissivity, being widely used for equipment thermal calibrations [22].

Some of the acquired screenshots are depicted in Figure 10 for three cases, i.e., nominal currents of $6 \mathrm{~A}, 10 \mathrm{~A}$, and $12 \mathrm{~A}$. For this experiment, five measurements have been performed and averaged for each power condition, thus providing improved accuracy. The junction temperature curves in Figure 11 compare the obtained values considering the indirect calculation method in (5) versus the experimental approximation via thermal camera addressed in this work. It is reasonable to state that the curves are nearly overlapped, thus validating the methodology employed to approximate $T_{j}$ experimentally. Besides, the aforementioned calibration technique has been satisfactorily able to provide an accurate $T_{j}$ estimation by the equipment.

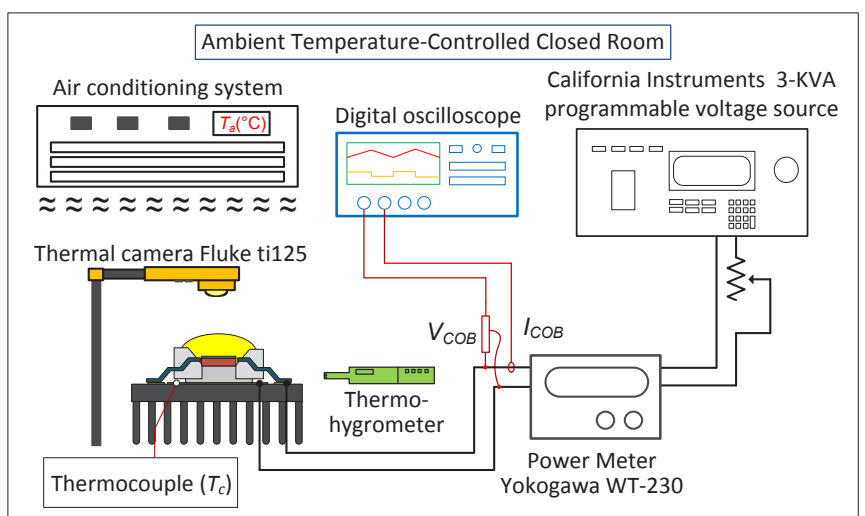

Fig. 9. Thermal-controlled experimental test for junction temperature measurements. 


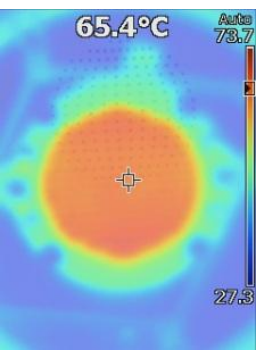

(a)

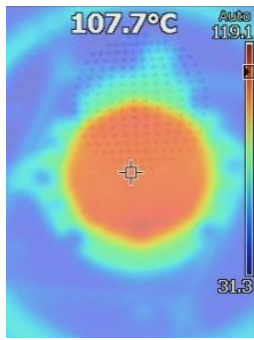

(b)

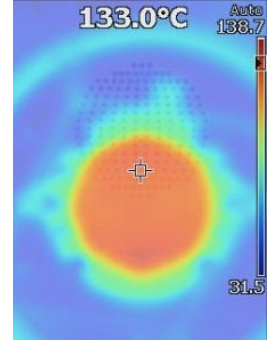

(c)
Fig. 10. Thermal camera screenshots for the experimenta approximation of the junction temperature. (a) $I_{C O B}=6 \mathrm{~A}$, (b) $I_{C O B}=$ $10 \mathrm{~A}$, and (c) $I_{C O B}=12 \mathrm{~A}$.

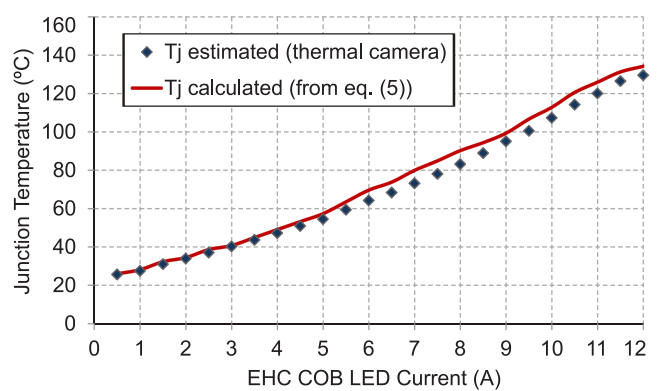

Fig. 11. Junction temperature as a function of the EHC COB LED current for Apollo 600: indirect calculation from (5) and approximate experimental estimation using a thermal camera.

The remaining parameters to be considered in the junction resistance calculation by (6) are the dissipated power $P_{d}$, which is measured by the power meter; and the heat dissipation factor $k_{h}$, which must be determined for each power point using (4). In this case, the experiment in Figure 12 has been employed, where an integrating sphere LMS-400 is used to measure the EHC COB LED radiant power for each power point. The resultant curve for $k_{h}$ coefficient variation is shown in Figure 13, where a second order polynomial approximation is performed to achieve a better fitting in this particular case. Therefore, the $k_{h}$ coefficient polynomial variation from (7) is also incorporated into the model, so that a better accuracy can be obtained. Considering the nominal current $(6 \mathrm{~A})$, the radiant efficiency and the heat dissipation coefficient are 0.388 and 0.612 , respectively. Since discrete low-current LEDs typically present radiant efficiencies close to $20 \%$ [8], the nominal value found for the studied device is very high, proving to be a prominent solidstate lighting technology.

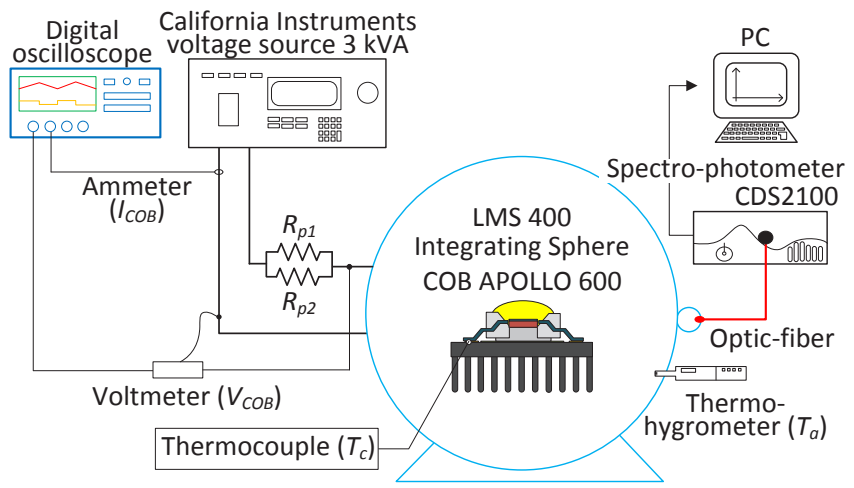

Fig. 12. Experimental setup employed in the reference luminous flux extraction and PET static model validation for Apollo 600.

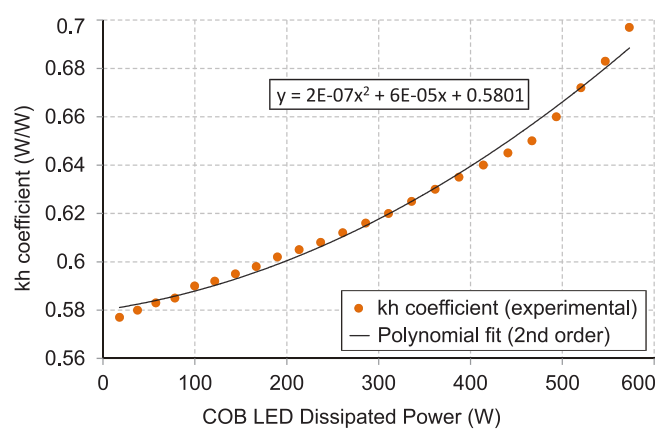

Fig. 13. Heat dissipation factor variation characteristic for Apollo 600 .

$$
k_{h}=k_{h 0}+k_{h 1} P_{d}+k_{h 2} P_{d}^{2}
$$

where $k_{h 0}, k_{h 1}$ and $k_{h 2}$ are the second-order polynomial coefficients associated to the heat dissipation.

From Figure 13, the heat dissipation coefficients are $k_{h 0}=0.5801 \mathrm{~W} / \mathrm{W}, k_{h 1}=6 \times 10^{-5} \mathrm{~W} / \mathrm{W}^{2}$, and $k_{h 2}=2 \times 10^{-7} \mathrm{~W} / \mathrm{W}^{3}$. Besides, the relationship in (6) is considered for point-topoint junction resistance calculation, while the obtained curve is shown in Figure 14. Thus, the linear variation of the junction resistance is defined as in (8), while its coefficients can be found as $k_{j c 0}=0.125^{\circ} \mathrm{C} / \mathrm{W}$ and $k_{j c 1}=0.0001{ }^{\circ} \mathrm{C} / \mathrm{W}^{2}$. From (8), one can notice that the very small value of $k_{j c 1}$ is multiplied by the high power of the EHC COB LED, thus influencing the real value of $R_{j c}$ significantly.

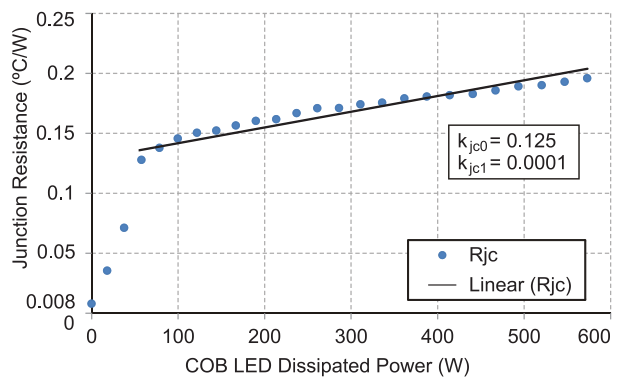

Fig. 14. Junction resistance variation characteristic for Apollo 600.

$$
R_{j c}=k_{j c 0}+k_{j c 1} P_{d}
$$

where $k_{j c 0}$ and $k_{j c 1}$ are the linear and slope coefficients, respectively.

The previously circuit in Figure 7 corresponds to the relationship between thermal and electrical domains, which can be analyzed to calculate the case temperature and also the junction temperature according to (9) and (10), respectively. The difference between (5) and (10) is the thermal dependence, which can be used to evidence the junction resistance thermal-dependent increment for the analyzed EHC COB device.

$$
T_{c}=T_{a}+Q_{t h} R_{h s}+Q_{t h} R_{t p}
$$

where $R_{t p}$ is the case-to-heatsink thermal paste resistance as provided by FCOpto $\left(R_{t p}=25 \times 10^{-6} \mathrm{C} / \mathrm{W}\right)$.

$$
T_{j}=T_{c}+\left(k_{j c 0}+k_{j c 1} P_{d}\right) Q_{t h} .
$$

The measured case temperatures from a thermometer with thermocouple in the experiment represented in Figure 9 can be used considering a constant value $R_{j c}=0.008{ }^{\circ} \mathrm{C} / \mathrm{W}$ provided in the datasheet or a linear variation of such parameter according to in Figure 14. Thus, the behavior of the device junction temperature will be also confirmed as a consequence. Figure 15 presents the measured case and 
junction temperatures calculated from (10) for both constant and linearly-varying $R_{j c}$ as a function of the forward current. The results show that the values obtained by considering $R_{j c}$ as given by (8) corroborate with the previous calculated and experimental estimations of $T_{j}$ in Figure 11. On the other hand, if $R_{j c}$ is considered constant, quite low and significantly mismatched values for $T_{j}$ are found. In fact, the experimental analysis of $k_{h}$ and $R_{j c}$ variations represent important directives to be considered in the COB technology, and thus a major contribution of this work. The highest percent instantaneous errors between the model and experiment for $T_{j}$ occur at high current levels. When comparing these calculated values of $T_{j}$ according to Figure 11, the linearlyvarying approach adopted for $R_{j c}$ returns an average percent error of only $2.2 \%$.

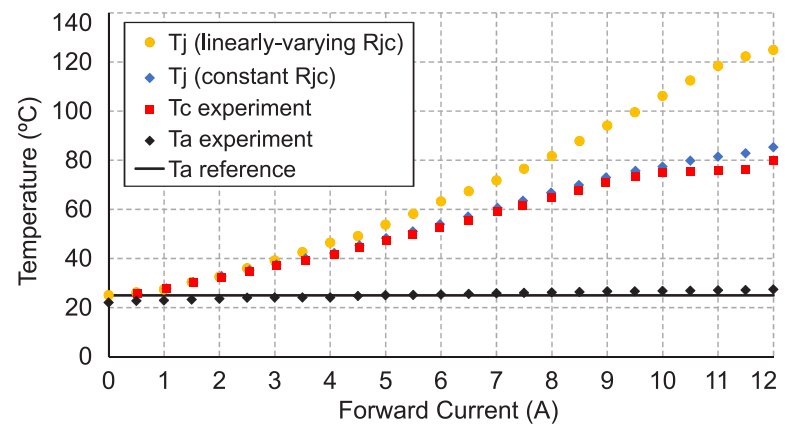

Fig. 15. Case, ambient, and calculated junction temperatures as a function of the EHC COB LED current: experimental and theoretical curves for Apollo 600.

Considering the previous model shown in Figure 7, an electrothermal mathematical representation can be also proposed while considering the thermal-related parameters variation. By combining expressions (2), (3), (9), and (10), it is possible to describe the thermal circuit for the forward voltage, resulting in (11).

$$
\begin{aligned}
& V_{\mathrm{COB}}\left(I_{\mathrm{COB}}, P_{d}, T_{a}\right)= \\
& \frac{V_{t}+r_{d} I_{C O B}+k_{v}\left(T_{a}-T_{0}\right)}{1-I_{C O B} k_{v}\left(k_{h 0}+k_{h 1} P_{d}+k_{h 2} P_{d}^{2}\right)\left(k_{j c 0}+k_{j c 1} P_{d}+R_{h s}+R_{t p}\right)}
\end{aligned}
$$

\section{Improved Photoelectrothermal (IPET) Model}

In order to aggregate all system interactions when dealing with the static model, it is necessary to establish a proper relationship between electrothermal and photometrical domains. Thus, two main photometrical characteristics are employed. Firstly, the photoelectrical curve in Figure 3.c represents the relationship between the luminous flux and current with constant junction temperature. Secondly, the photothermal curve in Figure 3.d details the interaction between luminous flux and junction temperature at a constant current. Such key parameters are not commonly found in technical datasheets, as they must be carefully analyzed and described experimentally.

The aforementioned curves are extracted to normalized luminous flux levels, as a flux of $100 \%$ corresponds both at the reference temperature $T_{0}$ and reference current $I_{0}$. The reference values are the ambient temperature $\left(25^{\circ} \mathrm{C}\right)$ and the current at the rated operating point (i.e., $6 \mathrm{~A}$ for Apollo 600). Linear approximations are performed for such curves, which considerably simplify the analysis. The normalized flux curves are approximated mathematically by (12) and (13), where $d_{0}, c_{0}$ and $d_{1}, c_{1}$ are their respective linear and angular coefficients. Coefficients $d_{0}$ and $d_{1}$ are assumed to be constant for any temperature value, while $c_{0}$ and $c_{1}$ are also constant for any current value [13].

$$
\begin{gathered}
\bar{\Phi}_{I}\left(I_{C O B}\right)=d_{0}+d_{1} I_{C O B} \\
\bar{\Phi}_{T}\left(T_{j}\right)=c_{0}+c_{1} T_{j} .
\end{gathered}
$$

The photoelectrical curve has been experimentally obtained using the arrangement shown in Figure 16. The photoelectrical coefficients $d_{0}$ and $d_{1}$ are not provided and must be obtained. This experiment consists in maintaining the room temperature at the reference value while $T_{c}$ is monitored by a thermocouple until the expected reference $\left(25^{\circ} \mathrm{C}\right)$ is reached. Current pulses are then applied starting from the maximum value $(12 \mathrm{~A})$ to the minimum one. The current pulses must be employed to ensure minimum variation between $T_{j}, T_{c}$ and $T_{a}$. A pulse width of $2 \mathrm{~ms}$ is employed in this test as seen in Figure 17. The system is assembled inside a black box, while a photodiode Vishay BPW21R (with lighting filter to prevent its saturation) and a precision transresistance amplifier are used to quantify the normalized luminous flux. The employed photodiode represents a light sensor whose sensibility curve is similar to that of the human eye, which presents fast response, i.e., rise time of $3 \mu \mathrm{s}$.

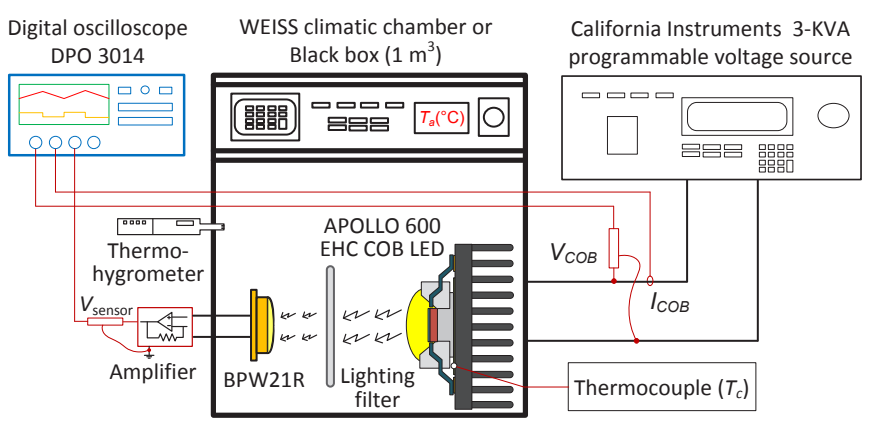

Fig. 16. Experimental setup employed for photo-related characterizations. Photoelectrical (black box) and photothermal (climatic chamber).

The photothermal analysis is not usually presented in EHC COB LEDs datasheets and it must be experimentally obtained from the arrangement shown in Figure 16 with the climatic chamber WEISS WKL-100/40. The photodiode is used once again to obtain the normalized luminous flux whereas care must be taken regarding the device saturation due to the high illuminance levels associated to Apollo 600. In this analysis, several values of the junction temperature must be considered and properly conditioned by the climatic chamber. The thermal steady-state behavior must be assumed for each measurement, which ensures $T_{j} \cong T_{a}$. Thus, the pulsed current must be applied in every measurement at the reference, i.e., $6 \mathrm{~A}$. An adequate pulse width is critical, as it must be large enough so that the electrical steady-state condition is achieved. This issue is particularly important as the voltage across the photodiode sensor is used to obtain the normalized luminous flux, which must be measured in steady-state condition. Also in this case, a pulse width of $2 \mathrm{~ms}$ is fair enough as seen in Figure 17. 
Figures 18 and 19 show the obtained photoelectrical and photothermal curves, respectively. Thus, the photometrical coefficients can be obtained as $d_{0}=0$ and $d_{1}=0.1653 \mathrm{~A}^{-1}$ from the photoelectrical curve; and $c_{0}=1.0478$ and $c_{1}=-0.0018^{\circ} \mathrm{C}^{-1}$ from the photothermal curve.

By combining the orthogonal expressions presented in (14), the IPET model for luminous flux prediction in EHC COB LEDs can be obtained as in (15), while modified to consider the main thermal parameters variation, i.e., $k_{h}$ and $R_{j c}$. Value $\Phi_{0}$ is the reference luminous flux, which is also not described in the device datasheet and must be found experimentally. This parameter is obtained under the reference current $(6 \mathrm{~A})$ and ambient temperature $\left(25^{\circ} \mathrm{C}\right)$. Inside the integrating sphere LMS-400 depicted in Figure 12 (previously presented), the reference current is applied in a pulse width equivalent to the spectro-photometer integrating time so that $T_{j}$ is maintained closer to $T_{a}$. Thus, the reference luminous flux for Apollo 600 can be found as 35,580 $\mathrm{lm}$.

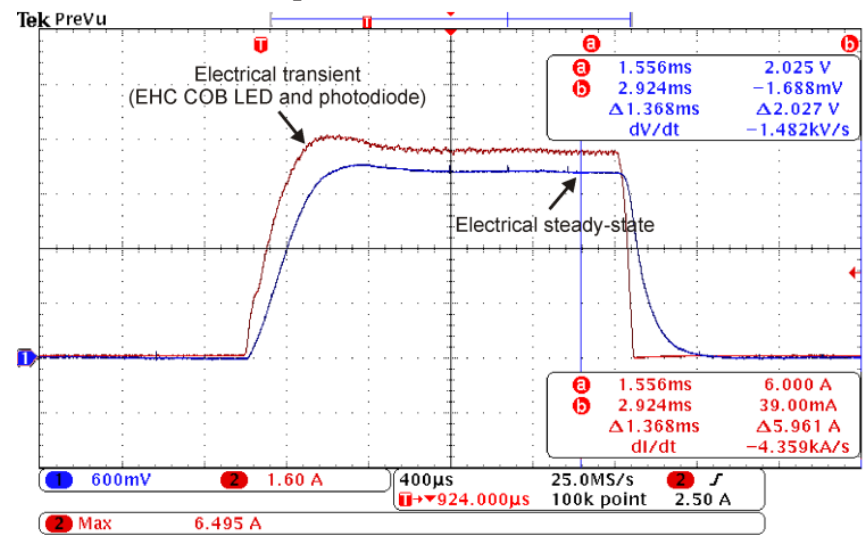

Fig. 17. EHC COB LED current pulse $(\mathrm{CH} 2-1.6 \mathrm{~A} /$ div. $)$ and photodiode voltage $(\mathrm{CH} 1-600 \mathrm{mV} /$ div.) waveforms with electrical steady-state acquiring methodology. Time scale: $400 \mu \mathrm{s} /$ div.

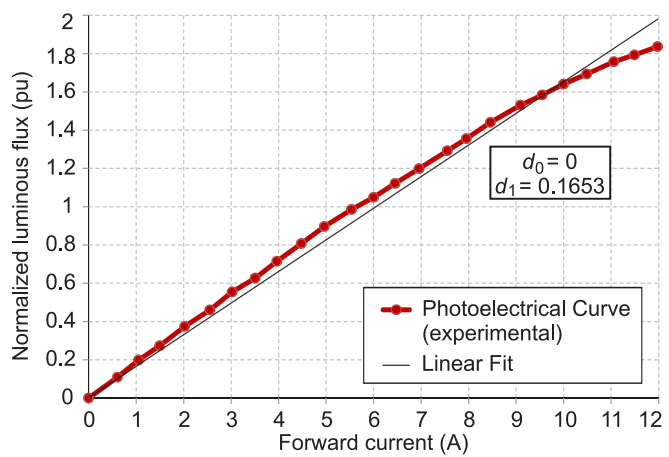

Fig. 18. Experimental photoelectrical characterization for Apollo $600\left(T_{0}=25^{\circ} \mathrm{C}\right)$

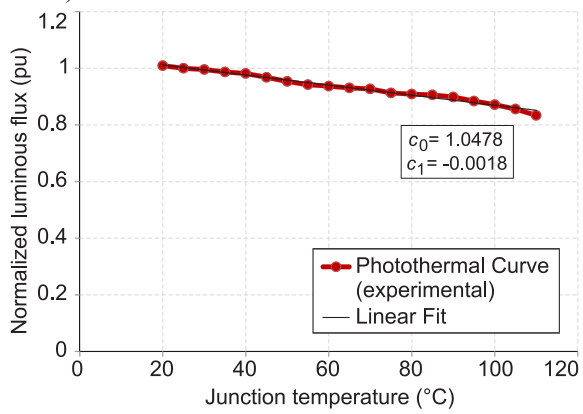

Fig. 19. Experimental photothermal characterization for Apollo 600 $\left(I_{0}=6 \mathrm{~A}\right)$.

$$
\begin{gathered}
\Phi_{C O B}=\Phi_{0}\left[\bar{\Phi}_{I}\left(I_{C O B}\right) \cdot \bar{\Phi}_{T}\left(T_{j}\right)\right] \\
\Phi_{C O B}=\Phi_{0}\left(d_{0}+d_{1} I_{C O B}\right)\left(c_{0}+c_{1} T_{j}\right) \\
\cdot\left\{c_{C O B}+c_{1}\left[\begin{array}{c}
T_{a}+\left(R_{j c}^{*}+R_{h s}+R_{t p}\right)\left(k_{h}^{*}\right) I_{C O B} \cdot \\
\cdot\left[\frac{V_{t}+r_{d} I_{C O B}+k_{v}\left(T_{a}-T_{0}\right)}{1-I_{C O B} k_{v}\left(k_{h}^{*}\right)\left(R_{j c}^{*}+R_{h s}+R_{t p}\right)}\right]
\end{array}\right]\right\}
\end{gathered}
$$

with: $k_{h}^{*}=k_{h 0}+k_{h 1} P_{d}+k_{h 2} P_{d}^{2}$

$$
R_{j c}^{*}=k_{j c 0}+k_{j c 1} P_{d}
$$

\section{EHC COB LED IPET MODEL EXPERIMENTAL VALIDATION AND DISCUSSION}

In order to experimentally validate the improved model, a laboratory setup has been implemented and analyzed. The test comprises Apollo 600 associated with a proper photospectrometer and integrated sphere (LMS-400), as depicted in the schematic shown in Figure 12. Besides, Table II summarizes all data collected from the previously described experiments. The results compare the performance of the theoretical model and the experimental setup. Figure 20 shows the curves regarding the luminous flux versus the direct current, while Figure 22 represents the curves of the luminous efficacy versus direct current. The voltage step corresponds to a variation of $0.5 \mathrm{~A}$ in the current, since twenty-four points have been acquired in the experimental evaluation.

TABLE II

Experimental PET Parameters Obtained For Apollo 600

\begin{tabular}{ccc}
\hline Parameter (Symbol) & Value & Unit \\
\hline Threshold voltage $\left(V_{t}\right)$ & 40.5 & $\mathrm{~V}$ \\
\hline Dynamic resistance $\left(r_{d}\right)$ & 0.95 & $\Omega$ \\
\hline Voltage thermal coefficient $\left(k_{v}\right)$ & -25.4 & $\mathrm{mV} /{ }^{\circ} \mathrm{C}$ \\
\hline Photoelectrical angular coefficient $\left(d_{0}\right)$ & 0 & - \\
\hline Photoelectrical linear coefficient $\left(d_{1}\right)$ & 0.1653 & $\mathrm{~A}^{-1}$ \\
\hline Photothermal angular coefficient $\left(c_{0}\right)$ & 1.0478 & - \\
\hline Photothermal linear coefficient $\left(c_{1}\right)$ & -0.0018 & ${ }^{\circ} \mathrm{C}^{-1}$ \\
\hline Heat dissipation coefficient a $\left(k_{h 2}\right)$ & $2 \times 10^{-7}$ & $\mathrm{~W} / \mathrm{W}^{3}$ \\
\hline Heat dissipation coefficient b $\left(k_{h 1}\right)$ & $6 \times 10^{-5}$ & $\mathrm{~W} / \mathrm{W}$ \\
\hline Heat dissipation coefficient c $\left(k_{h 0}\right)$ & 0.5801 & $\mathrm{~W} / \mathrm{W}$ \\
\hline Junction resistance angular coefficient $\left(k_{j c 0}\right)$ & 0.125 & ${ }^{\circ} \mathrm{C} / \mathrm{W}$ \\
\hline Junction resistance linear coefficient $\left(k_{j c 1}\right)$ & 0.0001 & ${ }^{\circ} \mathrm{C} / \mathrm{W}$ \\
\hline Case-to-heatsink thermal paste resistance $\left(R_{t p}\right)$ & 0.025 & $\mathrm{~m}^{\circ} \mathrm{C} / \mathrm{W}$ \\
\hline Reference luminous flux $\left(\Phi_{0}\right) @ 6 \mathrm{~A}, 25^{\circ} \mathrm{C}$ & 35580 & $1 \mathrm{~m}$ \\
\hline Radiant flux $\left(\Phi_{r a d}\right) @ 6 \mathrm{~A}, 25^{\circ} \mathrm{C}$ & 107.6 & $\mathrm{~W}$ \\
\hline
\end{tabular}

The luminous flux in Figure 20 presents parabolic behavior at high current values. This feature is related to the thermal resistances of the model, which is minimal for EHC COB LEDs. For low-current devices and their respective heatsinks, which generally present higher values of $R_{h s}$ and $R_{j c}$, this issue leads to more attenuated flux values at higher currents in the model curve [11]. For the studied EHC device, the constant value $R_{j c}=0.008{ }^{\circ} \mathrm{C} / \mathrm{W}$ provided in the datasheet is firstly adopted. The obtained average percent error when considering the model for which $R_{j c}$ is constant is found to be $6.3 \%$. The error increases above the nominal current point $(6 \mathrm{~A})$, since this firstly adopted model does not predict satisfactorily the flux attenuation at high current levels like in the extracted experimental points. 
Aiming at model improvement and reliability, the variations in thermal-related parameters, i.e., $k_{h}$ and $R_{j c}$, have been considered while the respective curve can be also seen in Figure 20. Considering this curve, the luminous flux prediction in high current levels is considerably improved, so that the estimation error remains lower than $2 \%$ when the device current is larger than $9 \mathrm{~A}$. The average percent error of the IPET model described by (15) is found to be $3.2 \%$, thus returning a great prediction of the luminous flux.

In order to provide a better insight about the improvement achieved by the proposed model, the instantaneous error curve tracked point to point for luminous flux prediction is presented in Figure 21. It is very important to highlight that the most important operating points in extra-high luminous flux applications are related to the highest current levels. Thus, the great accuracy of the employed technique can be noticed for extra-high current operating points. Considering the highest current, i.e., $12 \mathrm{~A}$, the instantaneous error reaches $16 \%$ for constant $R_{j c}$, and only $1.5 \%$ for the improved method as provided in this work. Yet, for a current point of $10.5 \mathrm{~A}$, the error related to the improved model is minimal, i.e., $0.3 \%$ in this case.

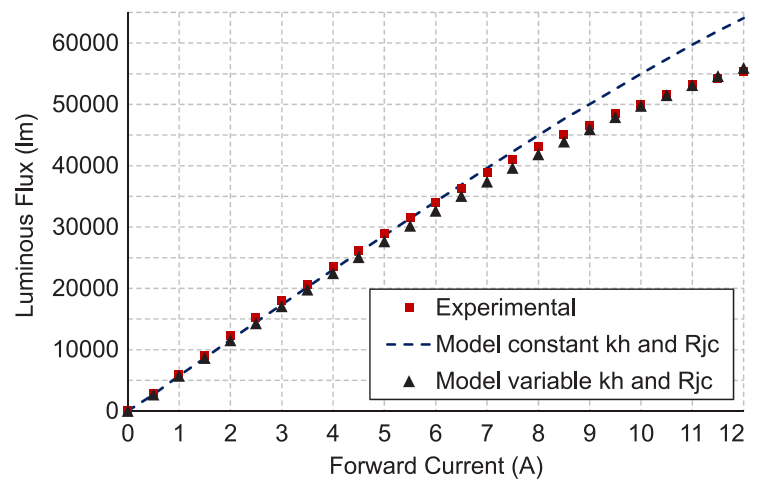

Fig. 20. Luminous flux as a function of the EHC COB LED current: experimental and theoretical curves for Apollo 600.

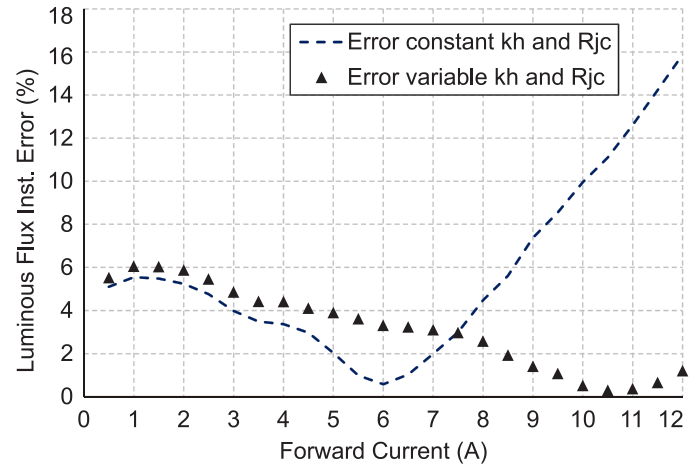

Fig. 21. Instantaneous errors for luminous flux prediction as a function of the EHC COB LED current.

According to Figure 22, the respective luminous efficacies are obtained from the ratio between luminous flux and active power for each measured point. Thus, from the extracted experimental points, the studied device is able to reach luminous efficacies rated at about $140 \mathrm{~lm} / \mathrm{W}$ at low current levels and $108 \mathrm{~lm} / \mathrm{W}$ at $10 \mathrm{~A}$. The model with constant thermal parameters attains an average error of $6.5 \%$. On the other hand, the improved model is able to predict the luminous efficacy with an average error of $3.4 \%$. Again, in order to analyze the remarkable improvement at the very high current points, the instantaneous errors tracked point to point for luminous efficacy are presented in Figure 23. Besides, the enhanced accuracy of the thermal variations model can be noticed for extra-high currents. For the highest point, i.e., $12 \mathrm{~A}$, the instantaneous error reaches $16.9 \%$ for the constant model, and only $1.8 \%$ for the improved method.

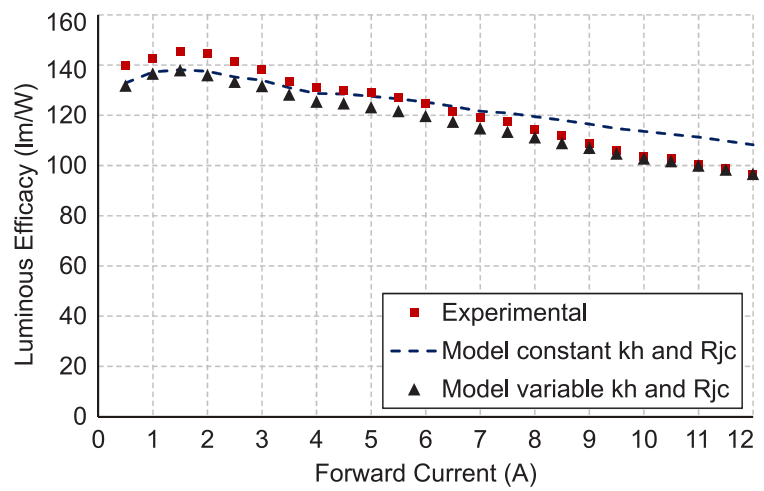

Fig. 22. Luminous efficacy as a function of the EHC COB LED current: experimental and theoretical curves for Apollo 600.

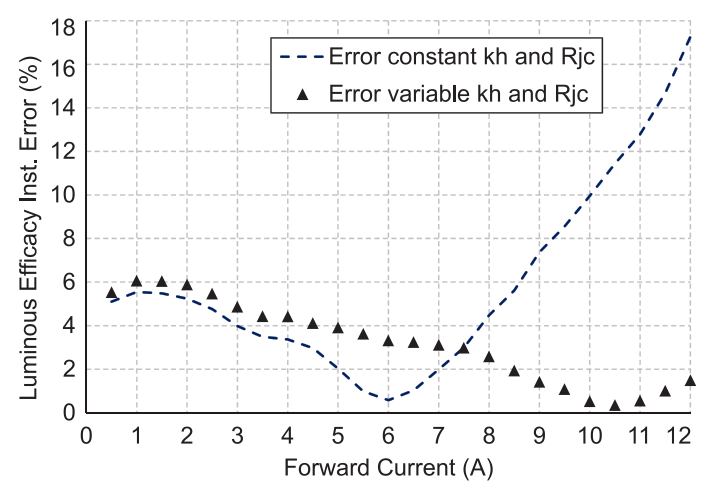

Fig. 23. Instantaneous errors for luminous efficacy prediction as a function of the EHC COB LED current.

The obtained photoelectrothermal profile of the lighting system is highly relevant in order to design a proper heatsink for an EHC COB LED, thus ensuring maximum luminous flux performance, minimized volume, and long lifespan at extra-high current levels [8]. By considering the derived IPET model represented by (15), a specific operation point must be chosen, and the related heatsink resistance can be accurately estimated as a consequence. This procedure ensures that the maximum luminous flux and the minimal heatsink thermal resistance can be obtained with consequent improved design in terms of the physical volume.

For instance, Figure 20 shows that the luminous flux corresponding to a current of $10 \mathrm{~A}$ is $49,758 \mathrm{~lm}$ when used with its respective $600-\mathrm{W}$ heatsink. Using the improved model with thermal variations represented by (15) in an application that requires a lower flux of $47,000 \mathrm{~lm}$ at the same current level, i.e., $10 \mathrm{~A}$, the calculated thermal resistance for heatsink designing could be increased from $0.0785{ }^{\circ} \mathrm{C} / \mathrm{W}$ (practical $600-\mathrm{W}$ heatsink) to $0.152{ }^{\circ} \mathrm{C} / \mathrm{W}$ (hypothetical $500-\mathrm{W}$ heatsink). Otherwise, when using the constant $k_{h}$ and $R_{j c}$ approach, the calculated $R_{h s}$ will be inaccurately oversized to $0,359{ }^{\circ} \mathrm{C} / \mathrm{W}$. Therefore, this value will correspond to a physically undersized and mismatched heatsink, thus leading to an inappropriate cooling system that highly degrades the EHC COB LED luminaire lifespan. 
To estimate the weight and cost of such improved heatsink, Table III presents an evaluative study regarding two heatsink models from Ursa Lighting/Starlite LED, i.e., up to $600-\mathrm{W}$ [17] and $320-\mathrm{W}$ [23], respectively, while also compared to a $500-\mathrm{W}$ theoretical heatsink. This model has been estimated by means of the aforementioned real devices physical parameters, while considering their weighted average values from (16). Thus, the size, weight and cost of the estimated $500-\mathrm{W}$ heatsink is significantly lower when compared to its $600-\mathrm{W}$ counterpart. Therefore, $R_{h s}$ values estimated from the proposed IPET model can be used in order to design optimized heatsinks for any operating point as desired, which includes especially, its extra-high current levels, where the proposed model attains remarkable accuracy for EHC COB LED applications.

$$
X_{W}=\frac{w_{1} x_{1}+w_{2} x_{2}}{w_{1}+w_{2}}
$$

where $X_{W}$ is the parameter weighted average value; $w_{1}=600 / 500$ and $w_{2}=320 / 500$ are the measuring weights for $600-\mathrm{W}$ and $320-\mathrm{W}$ heatsinks, respectively (i.e. their $500-\mathrm{W}$ related normalized values); $x_{1}$ and $x_{2}$ are the corresponding parameters for $600-\mathrm{W}$ and $320-\mathrm{W}$ heatsinks, respectively.

\section{TABLE III}

Comparative Analysis Regarding Cold Forged Heatsinks Employed to EHC COB LEDs

\begin{tabular}{cccc}
\hline Parameter & $\begin{array}{c}\mathbf{6 0 0 - W} \\
{[\mathbf{1 7 ]}}\end{array}$ & $\begin{array}{c}\mathbf{5 0 0 - W} \\
\text { (Estimated) }\end{array}$ & $\begin{array}{c}\mathbf{3 2 0 - W} \\
{[\mathbf{2 3}]}\end{array}$ \\
\hline Diameter $[\mathrm{mm}]$ & 341 & 305.8 & 240 \\
\hline Width $[\mathrm{mm}]$ & 150 & 150 & 150 \\
\hline Weight $[\mathrm{kg}]$ & 6.36 & 5.25 & 3.18 \\
\hline Amount of fins & 72 & 59 & 36 \\
\hline Thermal resistance $\left[{ }^{\circ} \mathrm{C} / \mathrm{W}\right]$ & 0.0785 & 0.152 & 0.2181 \\
\hline Estimated cost $[\mathrm{US} \$]$ & 200 & 158.2 & 80 \\
\hline
\end{tabular}

\section{CONCLUSIONS}

This paper has analyzed a novel technology of extra-high current COB devices, while the EHC COB LED Apollo 600 has been thoroughly studied. Great research potential can be addressed to modern EHC COB LEDs, which present distinct and challenging photoelectrothermal characteristics if compared with their low-current discrete counterparts.

In order to incorporate the heat dissipation factor and the junction resistance variations into a novel improved model, an experimental setup has been proposed. An experimental technique for the junction temperature approximate estimation has been proposed and compared to the indirect calculation method. The results have shown that the calculated and experimental techniques for $T_{j}$ estimation present minimal error, whereas the main thermal-related parameters, i.e., $k_{h}$ and $R_{j c}$, can be accurately obtained as a consequence. Besides, considering the theory allied to the experimental tests described in this work, EHC COB LED manufacturers can improve their datasheets following the reproducible directives.

An experimental setup has been also developed aiming at model validation. The average error between experiment and IPET model for luminous flux is $3.2 \%$, thus leading to an accurate prediction. This percentage for instantaneous errors is even lower for the highest current operating point, i.e.,
$1.5 \%$ for the proposed method, while the constant parameters method returns quite high and mismatched values, i.e., 16\%. The results show the critical influence of thermal-related parameters variations in $\mathrm{EHC} \mathrm{COB}$ devices, while the main contributions of this work can be summarized as:

1. The reproducible experimental methodology to achieve a concise static modeling for EHC COB LEDs, while obtaining all the related parameters which are not totally described in referred datasheets.

2. The incorporation of thermal parameters variations into a novel improved model, which greatly enhances the luminous flux prediction for very high current levels. This feature is particularly critical for the EHC COB technology due to its intrinsic and unique thermal characteristics. Methodologies are also described to estimate the device junction temperature, which has been validated considering mathematical and experimental approaches.

3. The experimental validation of the deduced IPET model, which shows the feasibility of the employed method. The instantaneous errors curves for luminous flux and efficacy show the accurate prediction of the proposed model, which presents minimal errors for the most important operating points in extra-high luminous flux applications, i.e., the highest current levels.

\section{ACKNOWLEDGEMENT}

The authors would like to acknowledge CAPES, CNPq, FAPEMIG, and INERGE for the financial support to this work; and Flip Chip Opto Company for the product and information support.

\section{REFERENCES}

[1] M. A. Reyes, J. J. Sammarco, S. Gallagher, and J. R. Srednicki. "Comparative evaluation of light-emitting diode lamps with an emphasis on visual performance in mesopic lighting conditions", in IEEE Transactions on Industry Applications, vol. 50, no. 1, pp. 127-133, Jan 2014.

[2] Osram, "Floodlight 20 Maxi LED Module Generation 2". Osram Lighting, 2018. Available in: https://goo.gl/wGmf33. Accessed in 01/30/2019.

[3] N. Kafadarova N. Vakrilov, A. Andonova. "Study of high power COB LED modules with respect to topology of chips", in Electronics Technology (ISSE), 38th International Spring Seminar on, vol. 2, pp. 108-113, May 2015.

[4] Flip Chip Opto, "FCOpto Starlite LED product catalogue", 2016. Available in: https://goo.gl/mxWZtx. Accessed in 02/03/2019.

[5] Flip Chip Opto, “Apollo 600 datasheet”, 2016. Available in: https://goo.gl/CxkdGi. Accessed in 02/03/2019.

[6] Lumileds, "Luxeon COB Core Range". Lumileds Lighting 2018. Available in: https://goo.gl/9F52Yh. Accessed in 02/03/2019.

[7] Bridgelux, "Décor Ultra High CRI Array Series". Bridgelux Lighting, 2018. Available in: https://goo.gl/wGqPn7. Accessed in 02/03/2019.

[8] R. Hui. Photo-electro-thermal Theory for LED Systems: Basic Theory and Applications, Cambridge University Press, 2017.

[9] Getian, "GT-P500W White COB LED Series". Getian Group, 2018. Available in: https://goo.gl/FYoKGd. Accessed in 01/30/2019.

[10] Yujileds, "BC270H High Power Density COB LED". Yujileds Lighting, 2018. Available in: https://goo.gl/HExJjz. Accessed in 01/30/2019. 
[11] S. Y Hui and Y. X Qin. "A general photo-electro-thermal theory for light emitting diode systems", in IEEE Transactions on Power electronics, vol. 24, no. 8, pp.19671976, Aug. 2009.

[12] V. C. Bender, O. Iaronka, W. D. Vizzotto, M. A. D. Costa, R. N. do Prado and T. B. Marchesan. "Design Methodology for Light-Emitting Diode Systems by Considering an Electrothermal Model", in IEEE Transactions on Electron. Devices, vol. 60, no. 11, pp. 3799-3806, Nov. 2013.

[13] P. S. Almeida, V. C. Bender, H. A. C. Braga, M. A. Dalla Costa, T. B. Marchesan, and J. M. Alonso. "Static and dynamic photoelectrothermal modeling of led lamps including low-frequency current ripple effects", in IEEE Transactions on Power Electronics, vol. 30, no. 7, pp. 3841-3851, July 2015.

[14] Philips, "Clear Flood Large LED Module BVP651 LED5004S/740 DM10 ALU PSU”. Philips Lighting, 2018. Available in: https://goo.gl/HWbpwf. Accessed in 01/30/2019.

[15] WEISS Technik, "Climatic Chamber WKL 100/40 model", 2010. Available in: https://goo.gl/p3bJEe. Accessed in $02 / 03 / 2019$.

[16] D. Gacio, J. M. Alonso, J. Garcia, M. S. Perdigão, E. S. Saraiva, F. E. Bisogno. "Effects of the junction temperature on the dynamic resistance of white LEDs", in IEEE Transactions on Industry Applications, vol. 49, no. 2, pp. 750760, Feb. 2013.

[17] Ursa Lighting/Starlite LED, "600-W Cold Forged Heatsink", 2016. Available in: https://goo.gl/LQ9RpN. Accessed in $01 / 30 / 2019$.

[18] J. Lalith, Y. M. Gu, and N. Nadarajah. "Characterization of thermal resistance coefficient of high-power LEDs", in Proc. 6th Int. Conf. Solid State Lighting, San Diego, CA, pp. 63370-63377, Aug. 2006.

[19] Z. L. Ma, X. R. Zheng, W. J. Liu, X. W. Lin, and W. L. Deng. "Fast thermal resistance measurement of high brightness LED", in Proc. 6th Int. Conf. Electron. Packag. Technol. (ICEPT 2005), Shenzhen, China, pp. 614-616, Aug. 2005.

[20] B. Siegal. "Practical considerations in high power LED junction temperature measurements", in Proc. 31st Int. Conf. Electron. Manuf. Technol. (IEMT 2006), Kuala Lumpur, Malaysia, pp. 62-66, Nov. 2006.

[21] I. U. Perera, N. Narendran, and Y. Liu. "Accurate measurement of LED lens surface temperature", in Proc. Int. Conf. Optical Engineering and Applications (SPIE 2013), San Diego, California, United States, pp. 1-6, Sep. 2013.

[22] H. Kaplan. Practical Applications of Infrared Thermal Sensing and Imaging Equipment, $3^{\text {rd }}$ ed., Int. Society of Optics and Photonics, Washington, 2007.

[23] Ursa Lighting/Starlite LED, "320-W Cold Forged Heatsink", 2016. Available in: https://goo.gl/6Rf7ah. Accessed in 01/30/2019.

\section{BIOGRAPHIES}

Dênis de C. Pereira received the B.Sc. and M. Sc. degrees in electrical engineering from the Federal University of São João del-Rei (UFSJ), Brazil, in 2013 and 2015, respectively. $\mathrm{He}$ is currently working towards the $\mathrm{Ph}$. D. degree in electrical engineering at the Federal University of Juiz de Fora (UFJF), Brazil. Since 2015, he has been a full-time researcher with the Modern Lighting Research Group (NIMO) at UFJF. His research interests include electronic power conversion, active power factor correction, solid-state lighting and LED driving, modeling and control of power electronic converters.

Pedro L. Tavares received the B.Sc. and M. Sc. degrees in electrical engineering from the Federal University of Juiz de Fora (UFJF), Brazil, in 2016 and 2018, respectively. He is currently working towards the $\mathrm{Ph}$. D. degree in electrical engineering. Since 2016, he has been a full-time research with the Modern Lighting Research Group (NIMO) at UFJF. His research interests include power electronic converters and general solid-state lighting.

Pedro S. Almeida received the B.Sc., M.Sc., and Ph.D. degrees in electrical engineering from the Federal University of Juiz de Fora (UFJF), Brazil, in 2010, 2012, and 2014, respectively. Since 2015, he has been a Professor of electrical engineering undergraduate and postgraduate courses at UFJF. His research interests include electronic power conversion, high power factor rectifiers and active power factor correction, solid-state lighting and LED driving, and modeling and control of electronic power converters.

Guilherme M. Soares received the B.Sc., M.Sc., and Ph.D. degrees in electrical engineering from the Federal University of Juiz de Fora (UFJF), Brazil, in 2012, 2014, and 2017, respectively. Since 2017, he has been a Professor with the Electrical Engineering Department, UFJF. His current research interests include electronic energy conversion, power factor correction, high reliability LED drivers, and general solid-state lighting.

Fernando L. Tofoli received the B.Sc., M.Sc., and Ph.D. degrees in electrical engineering from the Federal University of Uberlândia (UFU), Uberlândia, Brazil in 1999, 2002, and 2005, respectively. Since 2009, he has been a Professor with the Department of Electrical Engineering, Federal University of São João del-Rei (UFSJ), São João del-Rei, Brazil. His research interests include power-quality-related issues, highpower-factor rectifiers, non-isolated dc-dc converters with wide voltage conversion range, novel converter topologies, and solar photovoltaic systems.

Henrique A. C. Braga received the B.Sc. degree in electrical engineering from the Federal University of Juiz de Fora (UFJF), Juiz de Fora, Brazil, in 1982; the M.Sc. degree from Federal University of Rio de Janeiro (UFRJ), Rio de Janeiro, Brazil, in 1988; and the Ph.D. from the Federal University of Santa Catarina (UFSC), Florianópolis, Brazil, in 1996. He is currently a Full Professor with UFJF, where he teaches in the undergraduate and postgraduate programs in electrical engineering, mainly in topics regarding basic electronics and power electronics. From 2005 to 2006, he held a postdoctoral position at the University of Oviedo, Gijon, Spain. He is currently involved in several activities related to power electronics, efficient lighting, and power converters applied to renewable energy systems. Prof. Braga is a senior member of IEEE, and also a member of Brazilian Power Electronics Society. 